\title{
COMMENTARY
}

\section{Patient-ventilator trigger dys-synchrony: a common phenomenon with important implications}

\author{
Neil Maclntyre* \\ See related research by Carlucci et al., http://ccforum.com/content/17/2/R54
}

\begin{abstract}
Patient-ventilator trigger dys-synchronies are common with the use of assisted forms of mechanical ventilatory support, including non-invasive mechanical ventilatory support (NIV). Future system designs need to address this in order to improve the effectiveness of NIV.
\end{abstract}

Assisted/supported mechanical ventilation is designed to interact with patient muscle activity and 'share' the work of breathing. If properly done, assisted/supported ventilation unloads ventilatory muscles and reduces dyspnea. For this to occur, however, the ventilatory's flow and pressure delivery must synchronize with patient effort during all three phases of breath delivery: breath initiation, flow delivery, and breath termination (cycling). Dys-synchronous interactions can overload ventilatory muscles ('imposed' loads), compromise alveolar ventilation, overdistend alveolar units, disrupt sleep patterns, and cause patient discomfort, prompting additional sedation.

Carlucci and colleagues [1] have recently studied patient-ventilator interactions in 69 acutely ill patients receiving non-invasive ventilation (NIV) for a variety of reasons. They grouped these patients according to obstructive or restrictive pathophysiologies and carefully measured respiratory system mechanics (including esophageal pressure - a surrogate for pleural pressure). The patients were then initiated on NIV according to a standard protocol and were assessed for triggering dyssynchronies defined as missed triggers, delayed triggers, or double triggers. Importantly, they found that these

*Correspondence: neil.macintyre@duke.edu

Duke University Medical Center, Room 1120, Box 3911, Duke University Hospital, Durham, NC 27710 USA dys-synchronies were common (58\% of patients experienced them with missed triggers being the most frequent). Trigger dys-synchronies were associated with NIV intolerance and their prevalence was similar among the different patho-physiologic patterns.

This study is important as it underscores that patientventilator trigger dys-synchrony is common [2] and can be a major barrier to NIV effectiveness. This study also underscores the fact that the causes of trigger dyssynchrony are complex and affect all types of patients requiring assisted/supported mechanical ventilation [3-5]. Like invasive mechanical ventilation, NIV trigger dys-synchrony can stem from insensitive/poorly responsive breath triggering mechanisms. However, other factors also involved likely include ventilatory muscle loading patterns and their effects on the ventilatory controller, ventilatory muscle function, lung volumes at end-expiration (that is, intrinsic positive end-expiratory pressure (PEEP)), gas exchange effectiveness, and cortical inputs to the patient's ventilatory control center such as pain [3-5]. In addition, the triggering process with NIV can be further hampered by the mask system with its inherent leaks and claustrophobia exaggerating dyspnea and neural drive.

Importantly, this study did not address issues of flow synchrony or cycle synchrony, two other forms of patient ventilator interactions likely to impact NIV tolerance but not well studied in the current literature. Flow synchrony is defined as the ability of the ventilator to provide flow in accordance with patient demand as manifest by the ventilatory muscle contraction pattern. If flow is synchronous with that contraction pattern, the inspiratory muscle pressure time profile conceptually should resemble a near normal pattern. Note that flow synchrony does not mean the elimination of the work of breathing. Instead it means providing flow to 're-shape' the inspiratory muscle's pressure-time or pressure-volume profile to a more physiologic shape. In general, the variable flow features of pressure targeted breaths tend to flow 
synchronize easier with patient effort than clinician set flow-volume targeted breaths. This is why most NIV systems use pressure targeted breaths.

Cycle synchrony can also be important. An assisted/ supported mechanical breath termination shorter than the neural inspiratory time (machine $\mathrm{Ti}<$ neural $\mathrm{Ti}$ ) can lead to muscle activity beyond the machine's flow delivery phase, which can lead to high muscle loading, excessive tidal volumes and/or triggering of a second breath. In contrast, when mechanical breath cycling terminates after the inspiratory effort has ended (machine $\mathrm{Ti}>$ neural $\mathrm{Ti}$ ), dyspnea and expiratory muscle recruitment may occur in an effort to terminate the breath. Both can be addressed by altering breath cycling criteria.

Importantly, it is worth noting that dys-synchronous interactions often result in anxiety and dyspnea, which can stimulate overall ventilatory drive. Thus, improving synchrony in one area (for example, triggering) can help facilitate achieving synchrony in other areas (for example, flow demand).

In summary, patient-ventilator interactions are complex and are important to recognize and manage during both invasive and non-invasive mechanical ventilation. Carlucci and colleagues have demonstrated how common the trigger dys-synchronies are during NIV and that understanding them requires assessment not only of respiratory system mechanics, but also the complex interactions of neural drive, gas exchange, muscle function, and muscle loading. Much research is needed in this area as dys-synchronies of all types can lead to overloaded muscles and dyspneic patients requiring sedation - two factors that clearly can lead to prolonged mechanical ventilation days and poor outcomes.

\section{Abbreviations}

NIV, non-invasive ventilation

\section{Competing interests}

The author declares that he has no competing interests.

Published: 18 June 2013

\section{References}

1. Carlucci A, Pisani P, Ceriana P, Malovini A, Nava S: Patient ventilator asynchronies: may the respiratory system mechanics play a role? Crit Care 2013, 17:R54.

2. Epstein SK: How often does patient-ventilatory asynchrony occur and what are the consequences? Respir Care 2011, 56:25-38.

3. Pierson DJ: Conference Summary: Patient ventilator interactions. Resp Care 2011, 56:214-222.

4. Tobin MJ, Jubran A, Laghi F: Patient-ventilatory interaction. Am J Respir Crit Care Med 2001, 163:1059-1063.

5. Kondili E, Prinianakis G, Georgopoulos D: Patient-ventilator interaction. Br J Anaesth 2003, 91:106-119.

doi:10.1186/cc12729

Cite this article as: Maclntyre N: Patient-ventilator trigger dys-synchrony: a common phenomenon with important implications. Critical Care 2013, 17:157. 